Annales Academiæ Scientiarum Fennicæ

Mathematica

Volumen 36, 2011, 567-576

\title{
ON PLANAR HARMONIC LIPSCHITZ AND PLANAR HARMONIC HARDY CLASSES
}

\author{
Shaolin Chen, Saminathan Ponnusamy and Xiantao Wang* \\ Hunan Normal University, Department of Mathematics \\ Changsha, Hunan 410081, P. R. China; shlchen1982@yahoo.com.cn \\ Indian Institute of Technology Madras, Department of Mathematics \\ Chennai-600 036, India; samy@iitm.ac.in \\ Hunan Normal University, Department of Mathematics \\ Changsha, Hunan 410081, P. R. China; xtwang@hunnu.edu.cn
}

\begin{abstract}
In this paper, we investigate the properties of two classes of planar harmonic mappings. First, we discuss the equivalent norms on Lipschitz-type spaces of harmonic $K$-quasiregular mappings and then we study the relationship between the harmonic area functions and the harmonic Hardy classes. We also establish Landau's theorem for a class of harmonic Hardy mappings.
\end{abstract}

\section{Introduction and preliminaries}

A complex-valued function $f(z)=u(z)+i v(z)$ defined on a simply connected domain $D$ of $\mathbf{C}$ is called a harmonic mapping if and only if it is twice continuously differentiable and $\Delta f=0$. That is the components $u$ and $v$ are real harmonic in $D$, where $\Delta$ represents the complex Laplacian operator

$$
\Delta=4 \frac{\partial^{2}}{\partial z \partial \bar{z}}=\frac{\partial^{2}}{\partial x^{2}}+\frac{\partial^{2}}{\partial y^{2}}
$$

Every harmonic mapping $f$ defined in $D$ has a canonical decomposition $f=h+\bar{g}$, where $h$ and $g$ are analytic in $D$ (see [3] or [4]). Since the Jacobian $J_{f}(z)$ of $f$ is given by

$$
J_{f}(z)=\left|f_{z}(z)\right|^{2}-\left|f_{\bar{z}}(z)\right|^{2}=\left|h^{\prime}(z)\right|^{2}-\left|g^{\prime}(z)\right|^{2},
$$

$f$ is locally univalent and orientation-preserving if and only if $\left|g^{\prime}(z)\right|<\left|h^{\prime}(z)\right|$ in $D$; or equivalently $h^{\prime}(z) \neq 0$ and the dilatation $\omega=g^{\prime} / h^{\prime}$ has the property that $|\omega(z)|<1$ in $D$. For $a \in \mathbf{C}$, let $\mathbf{D}(a, r)=\{z:|z-a|<r\}$. In special, we denote $\mathbf{D}(0, r)=\mathbf{D}_{r}$ and $\mathbf{D}=\mathbf{D}(0,1)$. Because a composition $f \circ g$ with an analytic function $g$ remains harmonic, the Riemann mapping theorem allows us to assume that $D=\mathbf{D}$. Throughout this paper, we consider harmonic mappings in $\mathbf{D}$ unless specially stated.

A continuous increasing function $\omega:[0, \infty) \rightarrow[0, \infty)$ with $\omega(0)=0$ is called a majorant if $\omega(t) / t$ is non-increasing for $t>0$. Given a subset $\Omega$ of $\mathbf{C}$, a function $f: \Omega \rightarrow \mathbf{C}$ is said to belong to the Lipschitz space $\Lambda_{\omega}(\Omega)$ if there is a positive constant $C$ such that

$$
|f(z)-f(w)| \leq C \omega(|z-w|) \text { for all } z, w \in \Omega .
$$

doi:10.5186/aasfm.2011.3640

2010 Mathematics Subject Classification: Primary 30C65, 30C45; Secondary 30C20.

Key words: Harmonic quasiregular mapping, Lipschitz-type space, harmonic Hardy class, univalent functions, Landau's theorem.

*Corresponding author. 
For $\delta_{0}>0$, let

$$
\int_{0}^{\delta} \frac{\omega(t)}{t} d t \leq C \cdot \omega(\delta), \quad 0<\delta<\delta_{0}
$$

and

$$
\delta \int_{\delta}^{+\infty} \frac{\omega(t)}{t^{2}} d t \leq C \cdot \omega(\delta), \quad 0<\delta<\delta_{0}
$$

where $\omega$ is a majorant and $C$ is a positive constant. A majorant $\omega$ is said to be regular if it satisfies the conditions $(2)$ and $(3)$ (see $[5,16])$.

Let $G$ be a proper subdomain of $\mathbf{C}$ or $\mathbf{R}^{2}$. We say that a function $f$ belongs to the local Lipschitz space loc $\Lambda_{\omega}(G)$ if (1) holds, with a fixed positive constant $C$, whenever $z \in G$ and $|z-w|<\frac{1}{2} d(z, \partial G)$, where $d(\cdot, \cdot)$ denotes the Euclidean distance (cf. $[6,10]$ ). Moreover, $G$ is said to be a $\Lambda_{\omega}$-extension domain if $\Lambda_{\omega}(G)=\operatorname{loc} \Lambda_{\omega}(G)$. The geometric characterization of $\Lambda_{\omega}$-extension domains was first given by Gehring and Martio [6]. Then Lappalainen [10] extended it to the general case and proved that $G$ is a $\Lambda_{\omega}$-extension domain if and only if each pair of points $z, w \in G$ can be joined by a rectifiable curve $\gamma \subset G$ satisfying

$$
\int_{\gamma} \frac{\omega(d(z, \partial G))}{d(z, \partial G)} d s(z) \leq C \omega(|z-w|)
$$

with some fixed positive constant $C=C(G, \omega)$, where $d s$ stands for the arc length measure on $\gamma$. Furthermore, Lappalainen [10, Theorem 4.12] proved that $\Lambda_{\omega}$-extension domains exist only for majorants $\omega$ satisfying (2).

Dyakonov [5] characterized the holomorphic functions of class $\Lambda_{\omega}$ in terms of their modulus. Later in [16, Theorems A], Pavlović came up with a relatively simple proof of the results of Dyakonov. Recently, many authors considered this topic and generalized Dyakonov's results to pseudo-holomorphic functions and real harmonic functions of several variables for some special majorant $\omega(t)=t^{\alpha}$, where $\alpha>0$ (see $[9,11,12,13,14])$. In this paper, we first extend [16, Theorems A and B] to planar $K$-quasiregular harmonic mappings as follows, where $K \geq 1$.

Theorem 1. Let $\omega$ be a majorant satisfying (2), and let $G$ be a $\Lambda_{\omega}$-extension domain. If $f$ is a planar $K$-quasiregular harmonic mapping of $G$ and continuous up to the boundary $\partial G$, then

$$
f \in \Lambda_{\omega}(G) \Longleftrightarrow|f| \in \Lambda_{\omega}(G) \Longleftrightarrow|f| \in \Lambda_{\omega}(G, \partial G),
$$

where $\Lambda_{\omega}(G, \partial G)$ denotes the class of continuous functions $f$ on $G \cup \partial G$ which satisfy (1) with some positive constant $C$, whenever $z \in G$ and $w \in \partial G$.

For any $z_{1}, z_{2} \in G \subset \mathbf{C}$, let

$$
d_{\omega, G}\left(z_{1}, z_{2}\right):=\inf \int_{\gamma} \frac{\omega(d(z, \partial G))}{d(z, \partial G)} d s(z),
$$

where the infimum is taken over all rectifiable curves $\gamma \subset G$ joining $z_{1}$ to $z_{2}$. We say that $f \in \Lambda_{\omega, \inf }(G)$ whenever for any $z_{1}, z_{2} \in G$,

$$
\left|f\left(z_{1}\right)-f\left(z_{2}\right)\right| \leq C d_{\omega, G}\left(z_{1}, z_{2}\right),
$$

where $C$ is a positive constant which depends only on $f$ (see [8]). 
Theorem 2. Let $\omega$ be a majorant satisfying (2). If $f$ is a planar $K$-quasiregular harmonic mapping in $G$, then

$$
f \in \Lambda_{\omega, \inf }(G) \Longleftrightarrow|f| \in \Lambda_{\omega, \inf }(G) .
$$

Many authors discussed the relationships between Hardy classes of holomorphic functions and integral means (see [7, 15]). In order to derive an analogous result of [7, Theorem 1] for the setting of harmonic mappings, we need to introduce some notation.

For a harmonic function $f$ in $\mathbf{D}$, for $p>0$ and $0 \leq r<1$, we define

$$
I_{p}(r, f)=\frac{1}{2 \pi} \int_{0}^{2 \pi}\left|f\left(r e^{i \theta}\right)\right|^{p} d \theta
$$

and say that $f$ belongs to the harmonic Hardy class $\mathscr{H}_{h}^{p}$ if

$$
\|f\|_{p}=\sup _{0<r<1}\left(I_{p}(r, f)\right)^{1 / p}<+\infty .
$$

For a harmonic mapping $f$ in $\mathbf{D}$, the generalized harmonic area function $A_{h}(r, f)$ is defined by

$$
A_{h}(r, f)=\int_{\mathbf{D}_{r}}|\nabla f(z)|^{2} d A(z),
$$

where $d A$ denotes the normalized Lebesgue measure on $\mathbf{D}$ and

$$
|\nabla f|=\left(\left|f_{z}\right|^{2}+\left|f_{\bar{z}}\right|^{2}\right)^{1 / 2}
$$

The following theorem is an analogous result of [7, Theorem 1].

Theorem 3. Let $f$ be harmonic in $\mathbf{D}$ and $\delta>0$. Then, if $1<p \leq 2$,

$$
f \in \mathscr{H}_{h}^{p}(\mathbf{D}) \Rightarrow \int_{0}^{1} A_{h}^{\frac{p}{2}}(r, f)(1-r)^{\frac{\delta(2-p)}{2}} d r<+\infty,
$$

while if $p>2$,

$$
\int_{0}^{1} A_{h}^{\frac{p}{2}}(r, f)(1-r)^{\frac{\delta(2-p)}{2}} d r<+\infty \Rightarrow f \in \mathscr{H}_{h}^{p}(\mathbf{D})
$$

Theorem 4. Let $f \in \mathscr{H}_{h}^{p}(\mathbf{D})$ and $\delta>0$. If $1<p \leq 2$, then

$$
\lim _{r \rightarrow 1-}(1-r)^{\frac{\delta(2-p)+2}{p}} A_{h}(r, f)=0 .
$$

Finally, we prove a Landau's theorem for a class of harmonic Hardy mappings.

Theorem 5. Let $f$ be a harmonic in $\mathbf{D}$ with $\|f\|_{p} \leq M$ and $f(0)=\lambda_{f}(0)-1=0$, where $M$ is a positive constant, $\lambda_{f}(z)=|| f_{z}(z)|-| f_{\bar{z}}(z)||$ and $p \geq 1$. Then $f$ is univalent in $\mathbf{D}_{\rho_{0}}$, where

$$
\rho_{0}=\varphi\left(r_{0}\right)=\max _{0<r<1} \varphi(r), \quad \varphi(r)=r\left(1-\sqrt{\frac{t}{1+t}}\right),
$$

with

$$
t=\frac{4}{\pi} \cdot \frac{2^{\frac{1}{p}} M}{r(1-r)^{\frac{1}{p}}}
$$


Moreover, $f\left(\mathbf{D}_{\rho_{0}}\right)$ contains a univalent disk $\mathbf{D}_{R_{0}}$ with

$$
R_{0}=\frac{r_{0} \varphi\left(r_{0}\right)}{2 r_{0}-\varphi\left(r_{0}\right)} .
$$

In Theorem 5, we remark that $\max _{0<r<1} \varphi(r)$ does exist, since

$$
\lim _{r \rightarrow 0+} \varphi(r)=\lim _{r \rightarrow 1-} \varphi(r)=0 .
$$

The proofs of these theorems are presented in the following sections. We end this section with the following problem which is suggested by the referee: Does Theorem 1 still hold if the hypothesis "mappings being harmonic" is dropped?

\section{Harmonic Lipschitz classes}

In order to prove our main results, we need the following result.

Theorem A. [1, Theorem 7] If $f$ is a $K$-quasiregular harmonic mapping of $\mathbf{D}$ into itself, then

holds for $z \in \mathbf{D}$.

$$
\left|f_{z}(z)\right|+\left|f_{\bar{z}}(z)\right| \leq \frac{4 K}{\pi} \frac{\cos (|f(z) \pi / 2|)}{1-|z|^{2}}
$$

Proof of Theorem 1. The implications $f \in \Lambda_{\omega}(G) \Rightarrow|f| \in \Lambda_{\omega}(G) \Rightarrow|f| \in$ $\Lambda_{\omega}(G, \partial G)$ are obvious. We only need to prove $|f| \in \Lambda_{\omega}(G, \partial G) \Rightarrow f \in \Lambda_{\omega}(G)$. For a fixed point $z \in G$, let

$$
F(\eta)=f(z+d(z) \eta) / M_{z}, \quad \eta \in \mathbf{D}
$$

where $d(z):=d(z, \partial G)$ and $M_{z}:=\sup \{|f(\zeta)|:|\zeta-z|<d(z)\}$. By a simple calculation, we obtain that

$$
\frac{\left|F_{\eta}(\eta)\right|+\left|F_{\bar{\eta}}(\eta)\right|}{\left|F_{\eta}(\eta)\right|-\left|F_{\bar{\eta}}(\eta)\right|}=\frac{\left|f_{\xi}(\xi)\right|+\left|f_{\bar{\xi}}(\xi)\right|}{\left|f_{\xi}(\xi)\right|-\left|f_{\bar{\xi}}(\xi)\right|} \leq K,
$$

where $\xi=z+d(z) \eta$. Then $F$ is a $K$-quasiregular harmonic mapping of $\mathbf{D}$ into itself. By Theorem A, we have

$$
\left|F_{\eta}(0)\right|+\left|F_{\bar{\eta}}(0)\right| \leq \frac{4 K\left(1-|F(0)|^{2}\right)}{\pi}
$$

which in turn gives

$$
d(z)\left(\left|f_{\xi}(z)\right|+\left|f_{\bar{\xi}}(z)\right|\right) \leq \frac{8 K}{\pi}\left(M_{z}-|f(z)|\right)
$$

Without loss of generality, we let $\zeta \in \partial G$ with $|\zeta-z|=d(z)$, and let $w \in$ $\mathbf{D}(z, d(z))$. Then

$$
\begin{aligned}
|f(w)|-|f(z)| & \leq|| f(w)|-| f(\zeta)||+|| f(\zeta)|-| f(z)|| \\
& \leq C \omega(d(z))+C \omega(2 d(z)) \leq 3 C \omega(d(z))
\end{aligned}
$$

and so,

$$
\sup _{w \in \mathbf{D}(z, d(z))}(|f(w)|-|f(z)|) \leq 3 C \omega(d(z))
$$

which implies that

$$
M_{z}-|f(z)| \leq 3 C \omega(d(z))
$$


Thus, by (8) and (9), we have

$$
\left|f_{\xi}(z)\right|+\left|f_{\bar{\xi}}(z)\right| \leq \frac{24 C K}{\pi} \cdot \frac{\omega(d(z))}{d(z)}, \quad z \in G .
$$

Finally, given any two points $z_{1}, z_{2} \in G$, let $\gamma \subset G$ be a curve which joins $z_{1}, z_{2}$ satisfying (4). Integrating (10) along $\gamma$, we obtain that

$$
\left|f\left(z_{1}\right)-f\left(z_{2}\right)\right| \leq \int_{\gamma}\left(\left|f_{z}(z)\right|+\left|f_{\bar{z}}(z)\right|\right) d s(z) \leq \frac{24 C K}{\pi} \int_{\gamma} \frac{\omega(d(z))}{d(z)} d s(z) .
$$

Therefore, (4) and (11) yield

$$
\left|f\left(z_{1}\right)-f\left(z_{2}\right)\right| \leq C_{1} \cdot \omega\left(\left|z_{1}-z_{2}\right|\right),
$$

where $C_{1}$ is a positive constant. This completes the proof.

Proof of Theorem 2. The implication $f \in \Lambda_{\omega, \text { inf }}(G) \Rightarrow|f| \in \Lambda_{\omega, \text { inf }}(G)$ is obvious. We need only to prove that $|f| \in \Lambda_{\omega \text {,inf }}(G) \Rightarrow f \in \Lambda_{\omega \text {,inf }}(G)$. For a fixed point $z \in G$, let

$$
F(\eta)=f(z+d(z) \eta) / M_{z}, \quad \eta \in \mathbf{D}
$$

where $d(z):=d(z, \partial G)$ and $M_{z}:=\sup \{|f(\zeta)|:|\zeta-z|<d(z)\}$. From the proof of Theorem 1, it follows that

$$
d(z)\left(\left|f_{\xi}(z)\right|+\left|f_{\bar{\xi}}(z)\right|\right) \leq \frac{8 K}{\pi}\left(M_{z}-|f(z)|\right)
$$

where $\xi=z+d(z) \eta$. For $w \in \mathbf{D}(z, d(z))$, there exists a positive constant $C$ such that

$$
|f(w)|-|f(z)| \leq C d_{\omega, G}(w, z) \leq C \int_{[w, z]} \frac{\omega(d(\zeta, \partial G))}{d(\zeta, \partial G)} d s(\zeta),
$$

where $[w, z]$ denotes the straight segment with endpoints $w$ and $z$. We observe that if $\zeta \in[w, z]$, then one has $[w, z] \subset \mathbf{D}(z, d(z)) \subset G$ and therefore

$$
d(\zeta, \partial G) \geq d(\zeta, \partial \mathbf{D}(z, d(z)))
$$

which gives that

$$
\frac{\omega(d(\zeta, \partial G))}{d(\zeta, \partial G)} \leq \frac{\omega(d(\zeta, \partial \mathbf{D}(z, d(z))))}{d(\zeta, \partial \mathbf{D}(z, d(z)))} .
$$

For any $w \in \mathbf{D}(z, d(z)),(13)$ and (14) imply that

$$
\begin{aligned}
|f(w)|-|f(z)| & \leq C \int_{[w, z]} \frac{\omega(d(\zeta, \partial G))}{d(\zeta, \partial G)} d s(\zeta) \leq C \int_{[w, z]} \frac{\omega(d(\zeta, \partial \mathbf{D}(z, d(z))))}{d(\zeta, \partial \mathbf{D}(z, d(z)))} d s(\zeta) \\
& =C \int_{[w, z]} \frac{\omega(d(z)-|\zeta-z|)}{d(z)-|\zeta-z|} d s(\zeta) \leq C \int_{0}^{d(z)} \frac{\omega(t)}{t} d t \leq C \omega(d(z)) .
\end{aligned}
$$

From this we obtain that

$$
M_{z}-|f(z)| \leq C \omega(d(z)) .
$$

Again, for any $z_{1}, z_{2} \in G$, by (12) and (15), there exists a positive constant $C_{1}$ such that

$$
\left|f\left(z_{1}\right)-f\left(z_{2}\right)\right| \leq C_{1} d_{\omega, G}\left(z_{1}, z_{2}\right)
$$

and the proof of this theorem is completed. 


\section{Harmonic Hardy classes}

It is worth to remark that the standard technologies of analytic functions are not useful to prove Theorem 3 and therefore, we use Green's Theorem in its proof. Green's theorem states that if $g \in C^{2}(\mathbf{D})$, then

$$
\frac{1}{2 \pi} \int_{0}^{2 \pi} g\left(r e^{i \theta}\right) d \theta=g(0)+\frac{1}{2} \int_{\mathbf{D}_{r}} \Delta g(z) \log \left(\frac{r}{|z|}\right) d A(z), \quad 0 \leq r<1 .
$$

Proof of Theorem 3. First we prove the implication (7). Let $f \in \mathscr{H}_{h}^{p}(\mathbf{D})$ and $\delta>0$. For $0 \leq r<1$, by the Poisson integral formula, we have

$$
f(z)=\frac{1}{2 \pi} \int_{0}^{2 \pi} \frac{r^{2}-|z|^{2}}{\left|z-r e^{i \theta}\right|^{2}} f\left(r e^{i \theta}\right) d \theta, \quad z \in \mathbf{D}_{r} .
$$

Using Jensen's inequality, we have

$$
|f(z)|^{p} \leq \frac{1}{2 \pi} \int_{0}^{2 \pi} \frac{r^{2}-|z|^{2}}{\left|z-r e^{i \theta}\right|^{2}}\left|f\left(r e^{i \theta}\right)\right|^{p} d \theta \leq \frac{2 r I_{p}(r, f)}{r-|z|}
$$

and so

$$
|f(z)|^{p}(r-|z|) \leq 2 r I_{p}(r, f),
$$

where $I_{p}(r, f)$ is defined by (5). It follows that

$$
\int_{0}^{r}(r-\rho)^{\delta} M^{p}(\rho, f) d \rho \leq 2 r I_{p}(r, f) \int_{0}^{r} \frac{d \rho}{(r-\rho)^{1-\delta}} \leq \frac{2 r^{1+\delta} I_{p}(r, f)}{\delta},
$$

where $M(r, f)=\sup \{|f(z)|:|z|=r\}$. By (16), we have

$$
I_{p}(r, f)=|f(0)|^{p}+\frac{1}{2} \int_{\mathbf{D}_{r}} \Delta\left(|f(z)|^{p}\right) \log \frac{r}{|z|} d A(z)
$$

and therefore

$$
\begin{aligned}
r \frac{d}{d r} I_{p}(r, f)= & \frac{1}{2} \int_{\mathbf{D}_{r}} \Delta\left(|f(z)|^{p}\right) d A(z) \\
= & p \int_{\mathbf{D}_{r}}\left[\left(\frac{p}{2}-1\right)|f(z)|^{p-4} \mid f_{z}(z) \overline{f(z)}+f(z) \overline{\left.f_{\bar{z}}(z)\right|^{2}}\right. \\
& \left.\quad+|f(z)|^{p-2}|\nabla f(z)|^{2}\right] d A(z) \\
\leq & p(p-1) \int_{\mathbf{D}_{r}}|f(z)|^{p-2}|\nabla f(z)|^{2} d A(z) \\
\leq & p(p-1) M^{p-2}(r, f) \int_{\mathbf{D}_{r}}|\nabla f(z)|^{2} d A(z) \\
= & p(p-1) A_{h}(r, f) M^{p-2}(r, f) .
\end{aligned}
$$


By integration, Hölder's inequality and (18), we have

$$
\begin{aligned}
I_{p}(r, f) \leq|f(0)|^{p}+p(p-1) \int_{0}^{r} \frac{A_{h}(\rho, f)}{\rho} M^{p-2}(\rho, f) d \rho \\
\leq|f(0)|^{p}+p(p-1)\left[\int_{0}^{r}(r-\rho)^{\delta} M^{p}(\rho, f) d \rho\right]^{\frac{p-2}{p}} \\
\cdot\left[\int_{0}^{r}\left(\frac{A_{h}(\rho, f)}{\rho}\right)^{\frac{p}{2}}(r-\rho)^{\frac{\delta(2-p)}{2}} d \rho\right]^{\frac{2}{p}} \\
\leq|f(0)|^{p}+p(p-1)\left(\frac{2}{\delta}\right)^{\frac{p-2}{p}} I_{p}^{\frac{p-2}{p}}(r, f) \\
\cdot\left[\int_{0}^{r}\left(\frac{A_{h}(\rho, f)}{\rho}\right)^{\frac{p}{2}}(r-\rho)^{\frac{\delta(2-p)}{2}} d \rho\right]^{\frac{2}{p}} .
\end{aligned}
$$

Without loss of generality, we may now assume that $f(0) \neq 0$. Then we have

$$
I_{p}^{\frac{2}{p}}(r, f) \leq|f(0)|^{\frac{p^{2}-p+2}{p}}+p(p-1)\left(\frac{2}{\delta}\right)^{\frac{p-2}{p}}\left[\int_{0}^{r}\left(\frac{A_{h}(\rho, f)}{\rho}\right)^{\frac{p}{2}}(r-\rho)^{\frac{\delta(2-p)}{2}} d \rho\right]^{\frac{2}{p}}
$$

which shows that

$$
\int_{0}^{1} A_{h}^{\frac{p}{2}}(r, f)(1-r)^{\frac{\delta(2-p)}{2}} d r<+\infty \Rightarrow f \in \mathscr{H}_{h}^{p}(\mathbf{D}) .
$$

Next, we prove the implication (6). By a simple calculation, we get

$$
\begin{aligned}
r \frac{d}{d r} I_{p}(r, f)= & p \int_{\mathbf{D}_{r}}\left[\left(\frac{p}{2}-1\right)|f(z)|^{p-4}\left|f_{z}(z) \overline{f(z)}+f(z) \overline{f_{\bar{z}}(z)}\right|^{2}\right. \\
& \left.+|f(z)|^{p-2}|\nabla f(z)|^{2}\right] d A(z) \\
\geq & p(p-1) \int_{\mathbf{D}_{r}}|f(z)|^{p-2}|\nabla f(z)|^{2} d A(z)
\end{aligned}
$$

and

$$
A_{h}(r, f)=\int_{\mathbf{D}_{r}}|\nabla f(z)|^{2} d A(z) \leq M^{2-p}(r, f) \int_{\mathbf{D}_{r}}|f(z)|^{p-2}|\nabla f(z)|^{2} d A(z),
$$

which implies that

$$
p(p-1) A_{h}(r, f) \leq r \frac{d}{d r} I_{p}(r, f) M^{2-p}(r, f) .
$$

By (18) and Hölder's inequality, we see that

$$
\begin{aligned}
& {[p(p-1)]^{\frac{p}{2}} \int_{0}^{r}\left[\frac{A_{h}(\rho, f)}{\rho}(r-\rho)^{\frac{\delta(2-p)}{p}}\right]^{\frac{p}{2}} d \rho} \\
& \leq \int_{0}^{r}(r-\rho)^{\frac{\delta(2-p)}{2}} M^{\frac{p(2-p)}{2}}(r, f)\left(\frac{d}{d r} I_{p}(r, f)\right)^{\frac{p}{2}} d \rho \\
& \leq\left[\int_{0}^{r}(r-\rho)^{\delta} M^{p}(r, f) d \rho\right]^{\frac{2-p}{2}}\left(I_{p}(r, f)-I_{p}(0, f)\right)^{\frac{p}{2}} \\
& \leq\left(\frac{2}{\delta}\right)^{\frac{2-p}{2}} I_{p}(r, f),
\end{aligned}
$$


which yields

$$
f \in \mathscr{H}_{h}^{p}(\mathbf{D}) \Rightarrow \int_{0}^{1} A_{h}^{\frac{p}{2}}(r, f)(1-r)^{\frac{\delta(2-p)}{2}} d r<+\infty .
$$

The proof of the theorem is completed.

Proof of Theorem 4. It is not difficult to see that

$$
A_{h}^{\frac{p}{2}}(r, f) \int_{r}^{1}(1-\rho)^{\frac{\delta(2-p)}{2}} d \rho \leq \int_{r}^{1}(1-\rho)^{\frac{\delta(2-p)}{2}} A_{h}^{\frac{p}{2}}(\rho, f) d \rho
$$

which implies that

$$
\frac{2}{2+\delta(2-p)}(1-r)^{\frac{2+\delta(2-p)}{2}} A_{h}^{\frac{p}{2}}(r, f) \leq \int_{r}^{1}(1-\rho)^{\frac{\delta(2-p)}{2}} A_{h}^{\frac{p}{2}}(\rho, f) d \rho .
$$

By Theorem 3, we conclude that

$$
\int_{0}^{1}(1-\rho)^{\frac{\delta(2-p)}{2}} A_{h}^{\frac{p}{2}}(\rho, f) d \rho<+\infty
$$

which gives

$$
\lim _{r \rightarrow 1-}(1-r)^{\frac{\delta(2-p)+2}{p}} A_{h}(r, f)=0
$$

and the proof of the theorem is completed.

\section{Landau's theorem}

Proof of Theorem 5. By assumption and the inequaltiy (17), we have

$$
|f(z)| \leq \frac{2^{\frac{1}{p}} M}{(1-|z|)^{\frac{1}{p}}}, \quad z \in \mathbf{D} .
$$

Set $f(z)=\sum_{n=1}^{\infty} a_{n} z^{n}+\sum_{n=1}^{\infty} \bar{b}_{n} \bar{z}^{n}$, and, for $\zeta \in \mathbf{D}$, let $F(\zeta)=f(r \zeta) / r$ so that

$$
F(\zeta)=\sum_{n=1}^{\infty} A_{n} \zeta^{n}+\sum_{n=1}^{\infty} \bar{B}_{n} \bar{\zeta}^{n}
$$

where $A_{n}=a_{n} r^{n-1}$ and $B_{n}=b_{n} r^{n-1}$. Then $F(0)=\lambda_{F}(0)-1=0$ and

$$
|F(\zeta)| \leq \frac{2^{\frac{1}{p}} M}{r(1-r)^{\frac{1}{p}}}=M(r) \text { for } \zeta \in \mathbf{D}
$$

By $[2$, Lemma 1], for $n \in\{2,3, \cdots\}$, we have

$$
\left|A_{n}\right|+\left|B_{n}\right| \leq \frac{4 M(r)}{\pi}
$$

To prove the univalence of $F$, we choose two distinct points $\zeta_{1}, \zeta_{2} \in \mathbf{D}_{\rho_{1}(r)}$ and let $\zeta_{1}-\zeta_{2}=\left|\zeta_{1}-\zeta_{2}\right| e^{i \theta}$, where

$$
\rho_{1}(r)=1-\sqrt{\frac{t}{1+t}},
$$


with $t=\frac{4 M(r)}{\pi}$. Then (19) yields that

$$
\begin{aligned}
\left|F\left(\zeta_{2}\right)-F\left(\zeta_{1}\right)\right|= & \left|\int_{\left[\zeta_{1}, \zeta_{2}\right]} F_{\zeta}(\zeta) d \zeta+F_{\bar{\zeta}}(\zeta) d \bar{\zeta}\right| \\
\geq & \left|\int_{\left[\zeta_{1}, \zeta_{2}\right]} F_{\zeta}(0) d \zeta+F_{\bar{\zeta}}(0) d \bar{\zeta}\right| \\
& -\left|\int_{\left[\zeta_{1}, \zeta_{2}\right]}\left(F_{\zeta}(\zeta)-F_{\zeta}(0)\right) d \zeta+\left(F_{\bar{\zeta}}(\zeta)-F_{\bar{\zeta}}(0)\right) d \bar{\zeta}\right| \\
> & \left|\zeta_{1}-\zeta_{2}\right|\left[\lambda_{F}(0)-\sum_{n=2}^{\infty}\left(\left|A_{n}\right|+\left|B_{n}\right|\right) n \rho_{1}^{n-1}(r)\right] \\
\geq & \left|\zeta_{1}-\zeta_{2}\right|\left[1-\frac{4 M(r)}{\pi} \cdot \frac{\rho_{1}(r)\left(2-\rho_{1}(r)\right)}{\left(1-\rho_{1}(r)\right)^{2}}\right] \geq 0 .
\end{aligned}
$$

Here in the last step we use the fact that

$$
1-\frac{4 M(r)}{\pi} \cdot \frac{\rho_{1}(r)\left(2-\rho_{1}(r)\right)}{\left(1-\rho_{1}(r)\right)^{2}}=0 \text {, i.e. } \rho_{1}(r)=\frac{\varphi(r)}{r} .
$$

Thus, $F\left(\zeta_{2}\right) \neq F\left(\zeta_{1}\right)$. The univalence of $F$ follows from the arbitrariness of $\zeta_{1}$ and $\zeta_{2}$. This implies that $f$ is univalent in $\mathbf{D}_{r \rho_{1}(r)}$.

Now, for any $\zeta^{\prime}=\rho_{1}(r) e^{i \theta} \in \partial \mathbf{D}_{\rho_{1}(r)}$, we easily obtain that

$$
\begin{aligned}
\left|F\left(\zeta^{\prime}\right)\right| & \geq \rho_{1}(r)-\sum_{n=2}^{\infty}\left(\left|A_{n}\right|+\left|B_{n}\right|\right) \rho_{1}^{n}(r) \geq \rho_{1}(r)-\sum_{n=2}^{\infty} \frac{4 M(r)}{\pi} \rho_{1}^{n}(r) \\
& =\rho_{1}(r)\left[1-\frac{4 M(r)}{\pi} \frac{\rho_{1}(r)}{1-\rho_{1}(r)}\right]=\frac{R_{0}}{r},
\end{aligned}
$$

where the last step is a consequence of the expression for $\rho_{1}$ given by $\rho_{1}(r)=r^{-1} \varphi(r)$. Therefore, $f\left(\mathbf{D}_{r \rho_{1}(r)}\right)$ contains a univalent disk of radius $R_{0}$.

Acknowledgements. The authors would like to thank the referee for his (or her) careful reading of this paper and useful suggestions.

The research was partly supported by NSF of China (No. 11071063) and Hunan Provincial Innovation Foundation for Postgraduate (No. 125000-4113). The work was carried out while the first author was visiting IIT Madras, under "RTFDCS Fellowship". This author thanks Centre for Cooperation in Science \& Technology among Developing Societies (CCSTDS) for its support and cooperation. The research was also partly supported by the Program for Science and Technology Innovative Research Team in Higher Educational Institutions of Hunan Province.

\section{References}

[1] Chen, H.: The Schwarz-Pick lemma for planar harmonic mappings. - Sci. China Math. 54, 2011, doi:10.1007/s11425-011-4193-X.

[2] Chen, Sh., S. Ponnusamy, and X. Wang: Bloch and Landau's theorems for planar pharmonic mappings. - J. Math. Anal. Appl. 373, 2011, 102-110.

[3] Clunie, J. G., and T. Sheil-Small: Harmonic univalent functions. - Ann. Acad. Sci. Fenn. Ser. A I Math. 9, 1984, 3-25.

[4] Duren, P.: Harmonic mappings in the plane. - Cambridge Univ. Press, 2004. 
[5] Dyakonov, K. M.: Equivalent norms on Lipschitz-type spaces of holomorphic functions. Acta Math. 178, 1997, 143-167.

[6] Gehring, F. W., and O. Martio: Lipschitz-classes and quasiconformal mappings. - Ann. Acad. Sci. Fenn. Ser. A I Math. 10, 1985, 203-219.

[7] Holland, F., and J. B. Twomey: On Hardy classes and area function. - J. London Math. Soc. $17,1978,275-283$.

[8] Kaufman, R., and J. M. Wu: Distances and the Hardy-Littlewood property. - Complex Variables Theory Appl. 4, 1984, 1-5.

[9] KoJIĆ, V., and M. PAvlović: Subharmonicity of $|f|^{p}$ for quasiregular harmonic functions, with applications. - J. Math. Anal. Appl. 342, 2008, 742-746.

[10] Lappalainen, V.: Lip $h^{-}$-xtension domains. - Ann. Acad. Sci. Fenn. Ser. A I Math. Diss. 56, 1985.

[11] Mateljević, M.: A version of Bloch theorem for quasiregular harmonic mappings. -Rev. Roumaine Math. Pures Appl. 47, 2002, 705-707.

[12] Mateljević, M.: Versions of Koebe 1/4 theorem for analytic and quasiregular harmonic functions and applications. - Publ. Inst. Math. (Beograd) (N.S.) 84, 2008, 61-72.

[13] Mateljević, M.: Quasiconformal and quasiregular harmonic analogues of Koebe's theorem and applications. - Ann. Acad. Sci. Fenn. Math. 32, 2007, 301-315.

[14] Mateljević, M., and M. Vuorinen: On harmonic quasiconformal quasi-isometries. - J. Inequal. Appl. 2010:178732, 1-19, doi:10.1155/2010/1787.

[15] Noonan, J. W., and D. K. Thomas: The integral means of regular functions. - J. London Math. Soc. 9, 1975, 557-560.

[16] PAvlović, M.: On Dyakonov's paper Equivalent norms on Lipschitz-type spaces of holomorphic functions. - Acta Math. 183, 1999, 141-143.

Received 24 August 2010 